\title{
The Wrong Kind of Gravity
}

\author{
Wolfhard Janke ${ }^{1}$ and Desmond A. Johnston ${ }^{2}$ \\ ${ }^{1}$ Institut für Theoretische Physik, Universität Leipzig \\ D-04109 Leipzig, Germany \\ 2 Department of Mathematics, Heriot-Watt University \\ Edinburgh, EH144AS, Scotland
}

\begin{abstract}
The KPZ formula [1] shows that coupling central charge $c \leq 1$ spin models to $2 \mathrm{D}$ quantum gravity dresses the conformal weights to get new critical exponents, where the relation between the original and dressed weights depends only on $c$. At the discrete level the coupling to $2 \mathrm{D}$ gravity is effected by putting the spin models on annealed ensembles of $\Phi^{3}$ planar random graphs or their dual triangulations, where the connectivity fluctuates on the same time-scale as the spins.

Since the sole determining factor in the dressing is the central charge, one could contemplate putting a spin model on a quenched ensemble of 2D gravity graphs with the "wrong" $c$ value. We might then expect to see the critical exponents appropriate to the $c$ value used in generating the graphs. In such cases the KPZ formula could be interpreted as giving a continuous line of critical exponents which depend on this central charge. We note that rational exponents other than the KPZ values can be generated using this procedure for the Ising, tricritical Ising and 3-state Potts models.
\end{abstract}




\section{Introduction}

Among the many puzzles resolved by $2 \mathrm{D}$ conformal field theory is the appearance of rational critical exponents in models such as the $2 \mathrm{D}$ Ising and Potts models. The miracle is repeated when the models are coupled to 2D quantum gravity since, as was shown by KPZ [1] and DDK [2], the dressing of the conformal weights by the Liouville field of $2 \mathrm{D}$ quantum gravity leads to a new set of exponents which are nonetheless still rational. The key formula in establishing the relation between the bare $(\Delta)$ and dressed $(\tilde{\Delta})$ conformal weights

$$
\tilde{\Delta}=\frac{\sqrt{1-c+24 \Delta}-\sqrt{1-c}}{\sqrt{25-c}-\sqrt{1-c}}
$$

depends only on the central charge $c$ of the matter. The net effect of the gravitational dressing for the minimal $(p, q)$ conformal models with $c=1-$ $6(p-q)^{2} / p q$, where the primary scaling operators are labelled by two integers $r, s$ satisfying $1 \leq r \leq p-1,1 \leq s \leq q-1$, is to transmute the bare weights $\Delta_{r, s}=\Delta_{p-r, q-s}=\left[(r q-s p)^{2}-(p-q)^{2}\right] / 4 p q$ from the Kac table into

$$
\tilde{\Delta}_{r, s}=\frac{|r q-s p|-|p-q|}{p+q-|p-q|}
$$

where $|p-q|=1$ for unitary models. The relation between $\Delta$ and $\tilde{\Delta}$ may be written as

$$
\tilde{\Delta}-\Delta=-\frac{\xi^{2}}{2} \tilde{\Delta}(\tilde{\Delta}-1)
$$

where

$$
\xi=-\frac{1}{2 \sqrt{3}}(\sqrt{25-c}-\sqrt{1-c})
$$

and is called the KPZ scaling relation.

The effect of coupling various statistical mechanical models to 2D gravity, such as the Ising and $q \leq 4$ Potts models, can thus be calculated using

the KPZ/DDK results. If we denote the critical temperature for the phase transition in these models by $T_{c}$ and the reduced temperature $\left|T-T_{c}\right| / T_{c}$ 
by $t$ then the critical exponents $\alpha, \beta$ are defined in the standard manner as $t \rightarrow 0$ by

$$
\begin{aligned}
C_{\text {sing }} & \sim t^{-\alpha}, \\
M & \sim t^{\beta},
\end{aligned}
$$

where $C_{\text {sing }}$ is the singular part of the specific heat and $M$ is the magnetization. It is then possible to calculate $\alpha$ and $\beta$ using the conformal weights of the energy density operator $\Delta_{\epsilon}$ and spin operator $\Delta_{\sigma}$ in both the dressed and undressed cases,

$$
\begin{aligned}
\alpha & =\frac{1-2 \Delta_{\epsilon}}{1-\Delta_{\epsilon}}, \\
\beta & =\frac{\Delta_{\sigma}}{1-\Delta_{\epsilon}} .
\end{aligned}
$$

The various scaling relations between the critical exponents then allow the determination of the complete set of exponents.

\section{When the wrong $c$ can be right}

The preceding derivation is quite natural when considering the models in a gravitational context. Since the matter is interacting with gravity, it is the central charge of the matter itself which gets fed into the KPZ formula and returns the new set of rational dressed conformal weights and consequently new set of rational critical exponents. There are, however, circumstances in which one could conceive of coupling the conformal matter to graphs with the "wrong" central charge. The first of these is when one considers the matter living on a quenched ensemble of 2D gravity graphs, as was done in [3, 4]. In this case the interaction between the graphs and the matter is switched off and one is in effect looking at an ensemble with quenched connectivity disorder. This ensemble displays several interesting effects, including a softening of first-order phase transitions in $q>4$ Potts models to continuous transitions and the possible appearance of a new set of non-rational (but still algebraic) quenched exponents for $q \leq 4$ Potts models. In these respects it very much resembles the quenched bond disorder models that have attracted much attention recently [5] rather than other quenched connectivity disorder ensembles generated using Poisonnian random lattices [6, [] which retain the characteristics of their regular lattice counterparts. 
The relevant relation between the quenched dressed weights and bare weights is given by the $c=0$ version of the KPZ formula

$$
\tilde{\Delta}=\frac{\sqrt{1+24 \Delta}-1}{4} .
$$

It has recently been pointed out by Cardy [8] that one should, in fact, see multi-fractal scaling of local correlators on quenched gravity graphs, just as with quenched bond disorder. The $n$th power of a correlator with weight $\tilde{\Delta}$ averaged over the disorder scales not as $n \tilde{\Delta}$, but rather

$$
\tilde{\Delta}_{n}=\frac{\sqrt{1+24 n \Delta}-1}{4} .
$$

Freed from the bounds of using the "right" value of $c$ in the KPZ formula we can consider the effect of coupling conformal matter to other backgrounds, whether quenched or annealed. In the quenched case one is interested in calculating the (reduced) free energy $F=[\ln Z]_{a v}$ where $[\ldots]_{a v}$ is a quenched average over an ensemble of graphs characterized by a central charge $c=d$. Such graphs can be generated by using the adjacency matrix of the graph $G$ since the fixed area (i.e. fixed number of vertices) partition function $Z_{A}$ obtained on integrating out $d$ scalar fields with central charge $d$ is

$$
Z_{A}=\sum_{G \in \mathcal{G}(A)}\left(\operatorname{det} C_{G}\right)^{-d / 2},
$$

where $\mathcal{G}(\mathcal{A})$ is the class of graph being being summed over and $C_{G}$ is the adjacency matrix of the the graph $G$ :

$$
C_{G}= \begin{cases}q_{i} & \text { if } i=j \\ -n_{i j} & \text { if } i \text { and } j \text { are adjacent } \\ 0 & \text { otherwise. }\end{cases}
$$

Since $d$ is now a parameter, one can in principle use arbitrary negative or positive values in generating the ensemble of graphs. In the above $q_{i}$ is the order of vertex $i$ and $n_{i j}$ is the number of edges connecting the adjacent vertices $i$ and $j$, which can be more than one in certain ensembles $\mathcal{G}(\mathcal{A})$.

In analytical calculations for quenched ensembles we can use the replica trick to (formally) replace the free energy by the $n \rightarrow 0$ limit of an $n$ replicated version of our matter action

$$
\begin{aligned}
F & =\left[\lim _{n \rightarrow 0}\left(Z^{n}-1\right) / n\right]_{a v} \\
& =\lim _{n \rightarrow 0}\left(\left[Z^{n}\right]_{a v}-1\right) / n
\end{aligned}
$$


which relates the quenched ensemble to the annealed problem with $n$ copies of the matter fields of interest. The $[\ldots]_{a v}$ stands as before for a functional integral over surfaces with central charge $d$, now dynamically coupled to the matter fields. The total central charge is thus $c_{\text {total }}=d+n c_{\text {matter }}$ and in the quenched $n \rightarrow 0$ limit $c_{\text {total }} \rightarrow d$ is the number which appears in the KPZ formula. A simulation to this effect has already been carried out in [9] where good numerical agreement was obtained for the measured exponents of the Ising model on a quenched ensemble of $d=-5$ graphs and those calculated by substituting $c=d=-5$ in the KPZ formula for the dressed energy and spin weights 1 .

Since the central charge of the graphs is decoupled from the matter in quenched simulations such as that described above, the KPZ formula on such backgrounds can be thought of as giving a line of dressed conformal weights, say $\tilde{\Delta}(d)$, depending on the central charge associated with the graphs $d$. If we parameterise $d$ in the customary manner

$$
d=1-\frac{6(p-q)^{2}}{p q}
$$

we arrive at the following version of the KPZ formula

$$
\tilde{\Delta}(d)=\frac{\sqrt{p^{2}+q^{2}-2 p q(1-2 \Delta)}-|p-q|}{p+q-|p-q|}
$$

for the dressing of weights in a gravitational background characterised by central charge $d$. The energy and spin weights derived from this formula would then, via equ.(6), give a line of critical exponents $\alpha(d)$ and $\beta(d)$ which depended on the background central charge $d$. In annealed simulations the central charge of the (now non-replicated) matter should be included and $d$ is replaced by $c_{\text {total }}=d+c_{\text {matter }}$ in the above considerations.

\section{Rational Points}

On a line of continuously varying critical exponents the rational values are typically the most interesting, a prime example being the 8 vertex model on a

\footnotetext{
${ }^{1}$ The interpretation put upon the results there was that annealed and quenched ensemble of graphs gave the same results provided the total central charge, $c_{\text {total }}$, was the same - the difference is essentially semantic.
} 
square lattice where the Ising model, amongst others, appears at such a point. We might thus enquire whether rational points other than the standard ones (i.e. $d=1 / 2$ for Ising, $7 / 10$ for the tri-critical Ising model, $4 / 5$ for the 3 state Potts model) exist. In the case of the Ising model $\Delta_{\epsilon}=1 / 2, \Delta_{\sigma}=1 / 16$ and there are no other operators in the conformal table apart from the unit operator. If we want to obtain rational $\tilde{\Delta}_{\epsilon}(d)$ and $\tilde{\Delta}_{\sigma}(d)$, and hence rational $\alpha(d)$ and $\beta(d)$, we see from equ.(13) that both $p^{2}+q^{2}$ and $p^{2}+q^{2}-7 p q / 4$ must be perfect squares. The first condition will be satisfied by the first two members of any Pythagorean triple $(p, q, m)$ which can be parameterised in general as $p=u^{2}-v^{2}, q=2 u v, m=u^{2}+v^{2}$ with g.c.d. $(u, v)=1$. Inserting this into the second condition and looking at the possible factorisations shows that only two triples satisfy both conditions, $(3,4,5)$ and $(5,12,13)$. The first corresponds to $d=1 / 2$ and, as it should, returns the standard KPZ weights for the Ising model coupled to $2 \mathrm{D}$ gravity. The second, however, is a background with $d=-39 / 10$ and gives the weights

$$
\begin{aligned}
& \tilde{\Delta}_{\epsilon}\left(-\frac{39}{10}\right)=\frac{3}{5} \\
& \tilde{\Delta}_{\sigma}\left(-\frac{39}{10}\right)=\frac{1}{10}
\end{aligned}
$$

which translates to exponents $\alpha(-39 / 10)=-1 / 2, \beta(-39 / 10)=1 / 4$. For comparison we show in Table 1 the exponents $\alpha, \beta$ and $\gamma$ 药 for the flat lattice (Onsager exponents), KPZ $(d=1 / 2)$, quenched $(d=0)$ and $d=-39 / 10$.

\begin{tabular}{|c|c|c|c|}
\hline & $\alpha$ & $\beta$ & $\gamma$ \\
\hline Onsager & 0 & $\frac{1}{8}$ & $\frac{7}{4}$ \\
\hline KPZ $(d=1 / 2)$ & -1 & $\frac{1}{2}$ & 2 \\
\hline Quenched $(d=0)$ & -0.8685169 & 0.4167516 & 2.0350137 \\
\hline$d=-\frac{39}{10}$ & $-\frac{1}{2}$ & $\frac{1}{4}$ & 2 \\
\hline
\end{tabular}

Table 1: Critical exponents for the Ising model

Remarkably, $\alpha(-39 / 10)=-1 / 2$ is the standard KPZ value for the threestate Potts model which has $c=4 / 5$, but $\beta(-39 / 10)=1 / 4$ is half the

\footnotetext{
${ }^{2}$ Pythagorean triples are three integers satisfying $p^{2}+q^{2}=m^{2}$.

${ }^{3}$ Derived from the scaling relation $\alpha+2 \beta+\gamma=2$.
} 
3-state Potts model exponent. This prompts one to look at the weights of the three-state Potts model in their own right. In this case one has a much larger conformal grid of allowed scaling dimensions, twelve of which actually appear as physical operators. Demanding rationality for all of these turns out to be too restrictive for all but the KPZ values with $d=4 / 5$. However, if we ask for rationality of only the energy and spin operators, which have bare weights $\Delta_{\epsilon}=2 / 5$ and $\Delta_{\sigma}=1 / 15$ repectively, equ.(13) now shows that $p^{2}+q^{2}-(2 / 5) p q$ and $p^{2}+q^{2}-(26 / 15) p q$ must be perfect squares. We again find two possible solutions, $d=4 / 5$ and $d=-3886 / 1115$. The resulting exponents are tabulated in Table 2 along with the classical (fixed lattice), KPZ and quenched values.

\begin{tabular}{|c|c|c|c|}
\hline & $\alpha$ & $\beta$ & $\gamma$ \\
\hline Fixed & $\frac{1}{3}$ & $\frac{1}{9}$ & $\frac{13}{9}$ \\
\hline KPZ $(d=4 / 5)$ & $-\frac{1}{2}$ & $\frac{1}{2}$ & $\frac{3}{2}$ \\
\hline Quenched $(d=0)$ & -0.2932676 & 0.3511286 & 1.5910104 \\
\hline$d=-3886 / 1115$ & $-\frac{1}{27}$ & $\frac{6}{27}$ & $\frac{43}{27}$ \\
\hline
\end{tabular}

Table 2: Critical exponents for the 3-state Potts model

We have omitted the tricritical Ising model, which strictly speaking comes between the Ising and three-state Potts model in any classification, in our discussion. Once again demanding rationality for the full conformal grid is too restrictive to give any values of $d$ other than $7 / 10$, but we can still get rational values by restricting ourselves to rational energy and spin operator weights. In this case the bare weights are $\Delta_{\epsilon}=1 / 10$ and $\Delta_{\sigma}=3 / 80$ and we find a whole series of additional solutions $d=-1449 / 400,-3059 / 1430$, $-133763 / 156400, \ldots$ as well as (unlike the Ising and 3-state Potts models) positive solutions $d=69 / 70,44719 / 81200, \ldots$.

\section{Conclusions}

In summary, we have seen that treating the central charge in the KPZ formula as a free parameter admits rational exponent values other than the standard ones for Ising, tricritical Ising and three-state Potts models. The numerology is most convincing in the Ising case, since both the operators 
in its small conformal grid acquire rational weights when $d=-39 / 10$. Although rational weights can be arranged for the energy and spin operators at novel values of the central charge for the other two, the rest of the conformal grid still acquires irrational weights. The three-state Potts model has one other rational value of $d=-3886 / 1115$, and the tricritical Ising many, including positive values.

We have noted that feeding a value of the central charge other than that of the matter into the KPZ formula is precisely what is required when the matter/gravity back reaction is switched off, as in quenched simulations. Given the similarity of spin model behaviour on such ensembles to those with quenched bond disorder and the existence of non-perturbative results for the resulting exponents, investigation of such models may be useful for illuminating some of the murkier properties of ferromagnetic systems with quenched disorder. Formally exponents calculated for the "wrong" central charge values can also apply to annealed ensembles of graphs (i.e. the connectivity in the graphs is fluctuating on the same time-scale as the spins, even if the back reaction of the matter on the graphs is switched off) so long as the appropriate matter central charge is included.

Finally, it is worth noting that the new rational points all appear to be numerically accessible, since [9] investigated $c=-5$ and the central charges for the Ising and three-state Potts rational exponents are both in the vicinity of -4 . It would be interesting to investigate either numerically or analytically whether the rational points were simply a numerical accident, or differed in some manner from the rest of the line of exponents, as well as the occurrence of such points in other known models.

\section{Acknowledgements}

DJ was partially supported by a Royal Society of Edinburgh/SOEID Support Research Fellowship. The collaborative work of DJ and WJ was funded by ARC grant 313-ARC-XII-98/41.

\section{References}

[1] V.G. Knizhnik, A.M. Polyakov, and A.B. Zamolodchikov, Mod. Phys. Lett. A3 (1988) 819. 
[2] F. David, Mod. Phys. Lett. A3 (1988) 1651;

J. Distler and H. Kawai, Nucl. Phys. B321 (1989) 509.

[3] C.F. Baillie, K.A. Hawick, and D.A. Johnston, Phys. Lett. B328 (1994) 284.

[4] C.F. Baillie, W. Janke, and D.A. Johnston, Phys. Lett. B388 (1996) 14.

[5] J. Cardy, "Quenched Randomness at First-Order Transitions", invited talk at STATPHYS20, Paris, July 1998, published in: Physica A263 (1999) 215 [ cond-mat/9806355 ].

[6] W. Janke and R. Villanova, Phys. Lett. A209 (1995) 179.

[7] W. Janke and R. Villanova, Nucl. Phys. B (Proc. Suppl.) 47 (1996) 641; W. Janke, M. Katoot, and R. Villanova, Phys. Rev. B49 (1994) 9644.

[8] J. Cardy, J. Phys. A32 (1999) L177.

[9] K. Anagnostopoulos, P. Bialas, and G. Thorleifsson, "The Ising Model on a Quenched Ensemble of $c=-5$ Gravity Graphs" condmat/9804137. . 